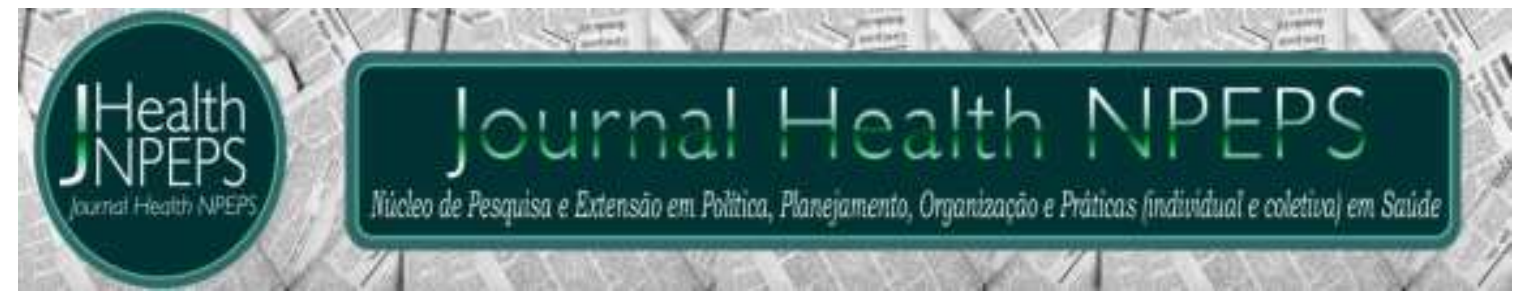

http://dx.doi.org/10.30681/252610104320

ARTIGO ORIGINAL

\title{
Apoyo social y participación en actividades recreativas en adultos mayores fronterizos
}

Social support and participation in recreational activities in border elderly

\section{Apoio social e participação em atividades recreativas em idosos fronteiriços}

\author{
Martha Elba Salazar-Barajas ${ }^{1}$, María Valentina Huerta-Gallegos ${ }^{2}$, Fernanda \\ Itzel Lankenau-Fuentes ${ }^{3}$, Ricardo Carmona-Carmona ${ }^{4}$, Tirso Duran-Badillo ${ }^{5}$, \\ Juana María Ruíz-Cerino ${ }^{6}$, Xóchitl Pérez-Zúñiga ${ }^{7}$, \\ Jesús Alejandro Guerra-Ordóñez ${ }^{8}$
}

\section{RESUMEN}

\begin{abstract}
${ }^{1}$ Doctora en Ciencias de Enfermería. Profesor de Tiempo Completo/Coordinador del GREEFES de la Unidad Académica Multidisciplinaria Matamoros. México. Email: msalazar@docentes.uat.edu.mx ORCID ID: https://orcid.org/0000-0001-6230-2547 Autor principal. Dirección de envío: Av del Maestro y Marte s/n col. Alianza CP.87400 Matamoros, Tamaulipas, México.

${ }^{2}$ Pasante de Licenciatura en Enfermería. Unidad Académica Multidisciplinaria Matamoros de la Universidad Autónoma de Tamaulipas. México. Email: valentinahuerta1@outlook.com ORCID ID: https://orcid.org/0000-0001-7998-1371

${ }^{3}$ Pasante de Licenciatura en Enfermería. Unidad Académica Multidisciplinaria Matamoros, de la Universidad Autónoma de Tamaulipas. México. Email: flankenau94@gmail.com ORCID ID: https://orcid.org/0000-0002-6554-5218

${ }^{4}$ Pasante de Licenciatura en Enfermería. Unidad Académica Multidisciplinaria de Matamoros, Universidad Autónoma de Tamaulipas. México. Email: riky carmona95@hotmail.com ORCID ID: https://orcid.org/0000-0001-5947-2211

${ }^{5}$ Doctor en Metodología de la Enseñanza. Profesor de Tiempo Completo/Coordinador de Titulación y Educación continua de la Unidad Académica Multidisciplinaria Matamoros. Universidad Autónoma de Tamaulipas. México. Email: tirdb27@hotmail.com ORCID ID: https://orcid.org/0000-0002-7274-3511 ${ }^{6}$ Doctora en Metodología de la Enseñanza. Profesor de Tiempo Completo/ Coordinadora de Laboratorio de Enfermería en la Unidad Académica Multidisciplinaria, Matamoros. Universidad Autónoma de Tamaulipas. México. Email: jruizc@docentes.uat.edu.mx ORCID ID: https://orcid.org/0000-0002-05412464

${ }^{7}$ Doctora en Metodología de la Enseñanza. Profesor Tiempo Completo de la Unidad Académica Multidisciplinaria de Matamoros de la Universidad Autónoma de Tamaulipas. México. Email: xperez@docentes.uat.edu.mx ORCID ID: https://orcid.org/0000-0002-2584-474X

${ }^{8}$ Doctor en Ciencias de Enfermería. Profesor de Tiempo Completo de la Unidad Académica Multidisciplinaria de Matamoros, Universidad Autónoma de Tamaulipas. México. Email: jesus.guerra@docentes.uat.edu.mx ORCID ID: https://orcid.org/0000-0002-4587-3214
\end{abstract}

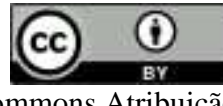

Este artigo está licenciado sob forma de uma licença Creative Commons Atribuição 4.0 Internacional, que permite uso irrestrito, distribuição e reprodução em qualquer meio, desde que a publicação original seja corretamente citada. 
Salazar-Barajas ME, Huerta-Gallegos MV, Lankenau-Fuentes FI, Carmona-Carmona R, Duran-Badillo T,

Objetivo: identificar la relación entre apoyo social y actividades recreativas en adultos mayores fronterizos. Método: diseño de tipo correlacional y transversal. Muestreo por conveniencia, con 110 adultos mayores de la ciudad de Matamoros, Tamaulipas. Se utilizó una cédula de datos personales, listado de actividades recreativas y el cuestionario de apoyo social funcional de DUKE-UNC-1. Resultados: la media de edad fue de 70.98 años (DE=6.54), el 99\% fueron mujeres. El acondicionamiento físico resulto la actividad más reportada (53.6). El apoyo social afectivo se observó en el 56.3\% mientras que el de confianza 51.8\%. El apoyo social se relacionó con las actividades recreativas $\left(r_{s}=.157, p<.05\right)$, y de forma negativa con la edad $\left(r_{s}=-.114\right.$, $p<.05)$, y a su vez las actividades recreativas no mostraron relación con la edad. Conclusión: el apoyo social y las actividades recreativas en el adulto mayor son determinantes para el mantenimiento de la salud física y mental, los resultados obtenidos muestran los beneficios de estas variables, por lo que profundizar en esta temática sería beneficioso para la disciplina de enfermería.

Descriptores: Apoyo Social; Actividades Recreativas; Adulto Mayor.

\section{ABSTRACT}

Objetive: to identify the relationship between social support and recreational activities in border elderly people. Method: correlational and transversal type design. Sampling for convenience, with 110 older adults from the city of Matamoros, Tamaulipas. A personal data card, a list of recreational activities and the DUKE-UNC-1 functional social support questionnaire were used. Results: the mean age was 70.98 years (SD $=6.54$ ) and $99 \%$ were women. Physical conditioning was the most reported activity (53.6). Affective social support was observed in 56.3\% while that of trust in 51.8\%. Social support is related to recreational activities ( $r s=.157, p<.05$ ), and negatively with age ( $r s=-.114, p<.05)$, and in turn, the recreational activities showed no relationship with the age. Conclusion: social support and recreational activities in the elderly are crucial for maintaining physical and mental health. The results obtained show the benefits of these variables, therefore, deepening this theme would be beneficial for the nursing discipline.

Descriptors: Social Support; Leisure Activities; Age.

\section{RESUMO}

Objetivo: identificar a relação entre apoio social e atividades recreativas em idosos fronteiriços. Método: desenho do tipo correlacional e transversal. Amostragem por conveniência, com 110 idosos da cidade de Matamoros, Tamaulipas. Foi utilizado um cartão de dados pessoais, uma lista de atividades recreativas e o questionário de suporte social funcional DUKE-UNC1. Resultados: a idade média de 70,98 anos ( $D P=6,54), 99 \%$ eram mulheres. 0 condicionamento físico foi a atividade mais relatada $(53,6)$. 0 suporte social afetivo foi observado em $56,3 \%$, enquanto o de confiança em $51,8 \%$. 0 apoio social está relacionado às atividades recreativas ( $r s=0,157, p<0,05$ ), e negativamente com a idade ( $r s=-114, p<0,05)$, e por sua vez as atividades recreativas não mostraram relação com a idade. Conclusão: o apoio social e as atividades recreativas em idosos são determinantes para a manutenção da saúde física e mental, os resultados obtidos mostram os benefícios dessas 
Salazar-Barajas ME, Huerta-Gallegos MV, Lankenau-Fuentes FI, Carmona-Carmona R, Duran-Badillo T,

variáveis, pois o aprofundamento desse tema seria benéfico para disciplina de enfermagem.

Descritores: Apoio Social; Atividades de Lazer; Idoso.

\section{INTRODUCCIÓN}

La organización mundial de la salud (OMS), menciona que la población ha envejecido desde el 2000, y actualmente mucho más rápido que años atrás, por lo que se espera que para el año 2050, la población de personas mayores aumente de un $11 \%$ al $22 \%$, es decir, pasara de 605 millones a 2000 millones en medio siglo ${ }^{1}$.

El consejo Nacional de Población, señaló que, México, será quien tenga la mayor población de adultos mayores (20.4\%) para el 2030, donde 57 de cada 100 adultos mayores, serán del sexo femenino y el 43 serán del sexo masculino, con un promedio de 70.9 años en población femenina y 70.4 en población masculina².

Una de las preocupaciones a nivel mundial y países en vías de desarrollo como es el caso de México, es lograr un envejecimiento activo, a su vez el envejecimiento es un proceso natural, sin embargo la forma de envejecer es diferente de una cultura a otra. El vivir muchos años es un logro gracias a los avances tecnológicos, la mejora en la atención a la salud, el control de la natalidad y el aumento de la esperanza de vida ${ }^{3}$. Por lo que el envejecimiento tiene que ver con visualizar esta etapa como en que las personas pueden seguir contribuyendo, desarrollando su potencial y ejerciendo sus derechos.

Por otro lado, con el envejecimiento ocurren cambios, debido a las modificaciones morfológicas, fisiológicas, bioquímicas y psicológicas propiciados por los cambios en la edad, por lo que un reto para la salud es retrasar la presencia de trastornos cognitivos y físicos para lograr mejores resultados de bienestar en los adultos mayores, sus familias y para la sociedad, a través de factores como el apoyo social y participación en actividades recreativas que contribuyan a un envejecimiento saludable ${ }^{4}$. 
Salazar-Barajas ME, Huerta-Gallegos MV, Lankenau-Fuentes FI, Carmona-Carmona R, Duran-Badillo T,

En el año 2002, la Organización Mundial de la Salud (OMS) convoco la II Asamblea Mundial del Envejecimiento en Madrid, donde definen el envejecimiento activo, como una forma de ver la vejez desde una perspectiva positiva y las características y factores que deben estar presentes 5 .

La literatura menciona que algunas características de un envejecimiento activo son, contar con la capacidad motora y cognitiva para poder realizar las actividades de la vida cotidiana de forma independiente y autónoma, realizar actividades deportivas, recreativas y culturales, además de participar en otras actividades que ayuden a integrarse con su entorno y mantener una vida productiva6.

Otros factores para un envejecimiento saludable son el estilo de vida, la alimentación saludable, realizar actividad física, evitar el consumo nocivo de alcohol, sustancias toxicas y tabaquismo ${ }^{1}$, además, poseer lazos afectivos familiares y de amistad, donde se brinden afecto, comprensión, respeto, y aceptación positiva del envejecimiento, satisfacción de necesidades cotidianas de recreación, acorde a las necesidades de la persona ${ }^{7}$.

En México se ha estudiado poco el apoyo social; se han reportado algunos casos de las relaciones sociales ${ }^{8}$ pero hasta el momento no se han encontrado estudios que reporten el apoyo social percibido y las actividades recreativas, estos conceptos son considerados de gran importancia para los adultos mayores, pero han sido poco explorados. El apoyo social es considerado como un soporte en estilos de vida más saludable y prevención de enfermedades mentales ${ }^{9}$. Una de forma de participar y relacionarse con los demás son las actividades recreativas y de ocio, deportivas, y culturales. La participación en diferentes actividades ayuda al adulto mayor a integrarse con su entorno y mantener una vida productiva $^{6}$.

El compromiso activo con la vida sugiere mantener relaciones de familia y con otras personas cercanas, además de realizar actividades significativas para ellos, componentes que incluyen 
Salazar-Barajas ME, Huerta-Gallegos MV, Lankenau-Fuentes FI, Carmona-Carmona R, Duran-Badillo T,

apoyo social y actividades recreativas.

El apoyo social es definido como una buena relación $e$ interacción con su entorno tanto familiar como dentro de la comunidad, que comprende lo psicológico, económico y social. Desde la perspectiva de la gerontología social, el apoyo social y las actividades recreativas al mismo de tiempo que ofrecen bienes a las personas que les rodean, pueden obtener beneficios y recompensas de la interacción con la sociedad ${ }^{10}$. Estos beneficios también pueden ser manejados como satisfacción para las necesidades emocionales que influyen para sentirse mejor fisca y emocionalmente. La relación entre apoyo social y la participación en actividades recreativas es compleja; autores incluso reconocen que puede ser bidireccional al incluir aspectos de salud.

Por otro lado, por actividades recreativas se entiende al conjunto de actividades agradables en las cuales se participa durante el tiempo libre y que fomentan el uso positivo de éste para promover el desarrollo integral de las personas. Las actividades pueden ser: acondicionamiento físico en todas sus formas como aerobics, zumba, deportes, caminatas; manualidades, bordado y tejido, corte y confección, música, guitarra, canto, danza y bailables, cocina y repostería, etc ${ }^{11}$. Es decir la participación en actividades recreativas significa compromiso consigo mismo y con otros.

Si las alteraciones de la salud debilitan las relaciones sociales y familiares, y las relaciones sociales débiles incrementan la enfermedad, se genera entonces un círculo vicioso $^{12}$. La relación del apoyo social y las actividades recreativas en adultos mayores es considerada indispensable por su naturaleza y contribuir en el entendimiento del fenómeno del envejecimiento.

Por lo tanto, el estudio del apoyo social percibido y la participación en actividades recreativas puede contribuir en el entendimiento del fenómeno de la funcionalidad de los adultos mayores. Así mismo, dado que no existe en el contexto 
Salazar-Barajas ME, Huerta-Gallegos MV, Lankenau-Fuentes FI, Carmona-Carmona R, Duran-Badillo T,

investigaciones similares, sirve de base para dar continuidad a investigaciones futuras. Por lo que el objetivo de este trabajo fue identificar la relación entre apoyo social y actividades recreativas en adultos mayores fronterizos.

\section{MÉTODO}

El diseño del estudio fue descriptivo, correlacional y transversal puesto que se pretendió encontrar relación entre apoyo social percibido y las actividades recreativas que realizan los adultos mayores. La población estuvo conformada por adultos mayores que asisten a la casa club del adulto mayor, de la ciudad fronteriza de Matamoros, Tamaulipas. El tamaño de la muestra se estimó a través del paquete Query Advisor 4.0, resultando en de 110 adultos mayores. Como criterios de inclusión se tomaron en cuenta adultos que tuvieran 60 años cumplidos y más, que pudieran escuchar, que estuvieran orientados en espacio y tiempo.

El muestro fue por conveniencia, primero se convocó a los adultos mayores a una reunión dentro de las instalaciones de la casa club, para dar a conocer el estudio, donde se explicó el objetivo y la importancia de participar y el tiempo probable de una hora. Se formuló una lista con nombres de aquellos decidieron aceptar, y posteriormente se acordó el día y la hora de reunión.

Para las mediciones se utilizó una cédula de datos personales que cuestionaba sobre edad, el sexo, años de estudio y estado civil de los participantes. En la misma cédula se incluyó un listado de nueve preguntas acordes con las mencionadas por la OMS y relacionadas como actividades recreativas para adultos mayores, entre ellas el acondicionamiento físico, bordado y tejido, cocina y repostería, coro y canto, corte y confección, danza y baile, manualidades, guitarra y jardinería y se agregó una pregunta adicional sobre otra actividad no mencionada. Las actividades en la casa club son 2 veces por semana de 1 hora semanal.

Para medir el apoyo social percibido se utilizó el cuestionario de apoyo social funcional de DUKE- 
Salazar-Barajas ME, Huerta-Gallegos MV, Lankenau-Fuentes FI, Carmona-Carmona R, Duran-Badillo T,

UNC-11Functional Social Support Questionnaire ${ }^{13}$. Es un cuestionario que tras ser validado en población mexicana $^{14}$ se redujo a dos dimensiones: afectivo y confianza. Tiene 6 preguntas sobre apoyo social afectivo $(1,4,6,7$ y 8$)$ y cinco para apoyo social de confianza (2, 3, 5, 9 y 11). Las puntuaciones mínimas y máximas por subescala oscilan entre 6 y 30 puntos para la primera y entre $5 \mathrm{y}$ 25 para la segunda.

De igual manera se estima que existe apoyo social afectivo si se obtiene un mínimo de 18 puntos y de confianza un mínimo de 15 puntos. Este cuestionario explora la cantidad de apoyo percibido, consta de 11 ítems, cada ítem consta de 5 respuestas, donde 1 es igual a mucho menos de lo que deseo, 2 menos de lo que deseo, 3 ni mucho ni poco, 4 más como lo deseo y 5 tanto como lo deseo. Este instrumento ha presentado buena consistencia con Alpha de Cronbach de 0.92. Los puntajes se transformaron a índice de 0 a 100 puntos.

Una vez seleccionados $e$ identificados los sujetos que participaron en la investigación, se les brindó un consentimiento informado por escrito y una vez firmados, los consentimientos fueron colocados en una caja y almacenados. Posteriormente se entregó la cédula datos personales, y listado de las actividades recreativas y finalmente el cuestionario de apoyo social

Una vez contestado, se agradeció a los participantes y los documentos fueron guardados en una caja independiente a los consentimientos, para su posterior captura en el programa Statistical Package for Social Sciences versión 22.0, donde se realizó estadística descriptiva e inferencial según el caso.

La investigación contó con la aprobación del comité de ética e investigación de la Unidad Académica Multidisciplinaria Matamoros de la Universidad Autónoma de Tamaulipas (073), además se apegó a lo establecido en la declaración de Helsinki sobre la promoción de la salud, bienestar y derechos del participante al no forzar su participación y evitar la presencia de situaciones $o$ procedimientos que le pudieran causar algún tipo de daño. Así 
Salazar-Barajas ME, Huerta-Gallegos MV, Lankenau-Fuentes FI, Carmona-Carmona R, Duran-Badillo T,

mismo, se protegió la dignidad, integridad, intimidad $y$ confidencialidad, al hacer un uso correcto de la información personal de los participantes.

Del mismo modo, la investigación fue realizada por profesionales con previa formación en el ámbito de la investigación, así como experiencia en la misma y el trato humano. Se informó acerca de los riesgos, costos y beneficios, donde estos últimos no eran representados en la investigación puesto que no genera costos ni beneficios percibidos para los participantes, únicamente acceso a la información una vez publicados los resultados. Respecto a los riesgos, se hizo de su conocimiento que participar no les generaba ningún riesgo. Se consideró a los participantes como grupo vulnerables debido a edad.

\section{RESULTADOS}

El coeficiente de Alpha de Cronbach del instrumento de apoyo social funcional fue de .88, el cual es aceptable de acuerdo a otros autores ${ }^{15}$. Dentro de los resultados del presente estudio en la Tabla 1 , se puede observar la distribución de frecuencia de las variables edad, escolaridad, apoyo social percibido y actividades recreativas. La prueba de Kolmogorov Smirnov con corrección de Lilliefors mostró que las distribuciones de frecuencia no siguieron una distribución normal.

Las frecuencias de variables sociodemográficas mostraron que la mayoría de los participantes correspondió al sexo femenino ( $f=99,90 \%$ ), la media de edad se situó en 70.9 años $(D E=6.54)$ con edades que oscilan entre 60 a 88 años. La escolaridad de 15 años y una media de 2.60 ( $D E=1.13)$. El $52.8 \%$ reporto primeria completa $(f=64)$ el $19.1 \% \quad(f=21)$ reporto haber terminado la secundaria y $16.3 \%$ el bachillerato y estudio técnico $(f=18)$, por último el 6.4 $(f=7)$ reporto no saber leer y escribir. 
Salazar-Barajas ME, Huerta-Gallegos MV, Lankenau-Fuentes FI, Carmona-Carmona R, Duran-Badillo T, Ruíz-Cerino JM, et al.

Apoyo social y participación en actividades recreativas...

Tabla 1- Características sociodemográficas y prueba de Kolgorov-Smirnov con corrección Lillefors.

\begin{tabular}{|c|c|c|c|c|c|c|c|}
\hline Variable & Media & $\overline{D E}$ & Mediana & $\begin{array}{l}\text { Valor } \\
\text { mínimo }\end{array}$ & $\begin{array}{l}\text { Valor } \\
\text { máximo }\end{array}$ & K_S & $\begin{array}{l}\text { Valor de } \\
p\end{array}$ \\
\hline Edad & 70.98 & 6.54 & 71.00 & 60 & 88 & .105 & .001 \\
\hline Escolaridad & 2.60 & 1.13 & 2.00 & 0 & 15 & .162 & .001 \\
\hline $\begin{array}{l}\text { Apoyo } \\
\text { social }\end{array}$ & 30.0 & 10.36 & 33.00 & 11 & 100 & .113 & .001 \\
\hline $\begin{array}{l}\text { Actividades } \\
\text { recreativas }\end{array}$ & 2.56 & 1.97 & 2.23 & 1 & 9 & .260 & .001 \\
\hline
\end{tabular}

Las actividades recreativas reportadas por los adultos mayores en la casa club tienen una duración aproximada de 1 hora, quienes reportaron más de una actividad, tomaban su clase un día diferente al señalado en la primera actividad. En la Tabla 2, se presentan las frecuencias de las mismas, se puede observar que la actividad mayormente realizada fue el acondicionamiento físico (53.6\%), en esta misma actividad el $12.7 \%$ realiza una segunda actividad a la semana y casi la mitad de los participantes señalo realizar manualidades (47.2\%).

Tabla 2 - Actividades recreativas de los adultos mayores.

\begin{tabular}{|c|c|c|c|c|c|c|}
\hline \multirow[t]{2}{*}{ Actividad } & \multicolumn{2}{|c|}{ Si } & \multicolumn{2}{|c|}{1 actividad } & \multicolumn{2}{|c|}{2 actividades } \\
\hline & $f$ & $\%$ & $f$ & $\%$ & $f$ & $\%$ \\
\hline Acondicionamiento físico & 59 & 53.6 & 45 & 40.9 & 14 & 12.7 \\
\hline Bordado / Tejido & 46 & 41.8 & 35 & 31.8 & 11 & 10 \\
\hline Cocina / repostería & 37 & 33.6 & 32 & 29.0 & 5 & 4.5 \\
\hline Coro / Canto & 16 & 14.5 & 0 & 0 & 16 & 14.5 \\
\hline Corte / confección & 18 & 16.3 & 7 & 6.36 & 11 & 10 \\
\hline Danza /baile & 27 & 24.5 & 11 & 10 & 16 & 14.5 \\
\hline Manualidades & 52 & 47.2 & 38 & 34.5 & 14 & 12.7 \\
\hline Guitarra & 15 & 13.6 & 8 & 7.27 & 3 & 2.72 \\
\hline Jardinería & 28 & 25.4 & 28 & 25.4 & 0 & 0 \\
\hline
\end{tabular}

Nota: $f=$ frecuencia $; \%=$ Porcentaje $n=110$

Los resultados del apoyo social percibido por los participantes se presentan en afectivo y de confianza. El apoyo social afectivo se observó en el $56.3 \%(f=62)$ de los participantes, 
Salazar-Barajas ME, Huerta-Gallegos MV, Lankenau-Fuentes FI, Carmona-Carmona R, Duran-Badillo T,

mientras que el de confianza se presentó en menor proporción $51.8 \%(f=57)$.

El apoyo social de tipo afectivo con mayor frecuencia fue "contar con personas que se preocupan por lo que me pasa" y "hablar con alguien sobre problemas familiares y personales" $(60 \% ; f=66)$. Mientras que el apoyo social del tipo confianza sobresalió "el recibir amor y afecto" con un $60.9 \%(f=67)$. Mientras que el de "recibir invitaciones para socializar solo estuvo presente en el $17.5 \%$ $(f=35)$.

En la Tabla 3 se presentan datos descriptivos de las sub escalas de apoyo social.

Tabla 3 - Tipos de apoyo social.

\begin{tabular}{lllllll}
\hline $\begin{array}{l}\text { Apoyo } \\
\text { social }\end{array}$ & Media & Mediana & DE & Varianza & Mínimo & Máximo \\
\cline { 2 - 7 } $\begin{array}{l}\text { afectivo } \\
\begin{array}{l}\text { Apoyo } \\
\text { social de } \\
\text { confianza }\end{array}\end{array}$ & 3.40 & 4.00 & 1.448 & 2.098 & 1 & 5 \\
$\begin{array}{l}\text { Nota: } n=110 ; \\
\text { DE }\end{array}$ & 3.00 & 1.524 & 2.324 & 1 & 5 \\
\hline
\end{tabular}

Tabla 4 - Correlación de Spearman de apoyo social, actividades recreativas y edad.

\begin{tabular}{lccc}
\hline \multicolumn{1}{c}{ Variable } & 1 & 2 & 3 \\
\hline 1.Edad & 1 & & \\
2.Apoyo social & $-.114^{*}$ & 1 & 1 \\
3.Actividades & .045 & $.157^{*}$
\end{tabular}

Nota: $n=110 ;{ }^{*}=p<.05$

Para dar respuesta al objetivo planteado se realizó correlación de Spearman, en la tabla 4, se presentan las relaciones y se puede observar que el apoyo social se relaciona con las actividades recreativas $\left(r_{s}=.157\right.$, $p<.05)$, y de forma negativa con la edad $\left(r_{s}=-.114, p<.05\right)$, y a su vez las actividades recreativas no mostraron relación con la edad.

\section{DISCUSIÓN}

La media de edad de los participantes fue casi de 71 años, datos que coinciden con otro estudio $^{9}$ y que reflejan que la participación de este grupo de edad en los estudios de investigación es muy activa. Otro dato que llama la atención en este estudio es la prevalencia de participación de las mujeres, resultados afines al estudio de 
Salazar-Barajas ME, Huerta-Gallegos MV, Lankenau-Fuentes FI, Carmona-Carmona R, Duran-Badillo T,

Sánchez-Padilla ${ }^{16}$, sin embargo a este respecto podemos decir que probablemente la participación en una casa club es más habitual la asistencia de mujeres que de hombres por las actividades que se ofertan.

Un hallazgo que llama la atención en los resultados presentados es que, la escolaridad presentó una media de 2.6 años, casi la mitad de los participantes reportaron el porcentaje de escolaridad en este rubro. Estos resultados encontrados están muy por debajo de la media Nacional que es de 9.2 en promedio ${ }^{17}$. No se investigó en los participantes si eran originarios de esta zona fronteriza o provenían de otro lugar de la republica u otros países, Matamoros es una zona fronteriza y actualmente con población flotante en espera de cruzar a los Estados Unidos de América.

La actividad física consiste en actividades recreativas o de ocio que incluye paseos, caminatas, actividades ocupacionales (trabajos) tareas domésticas, juegos deporte o ejercicio programado ${ }^{18}$. Al respecto se puede mencionar que el $100 \%$ de la muestra menciono realizar al menos una actividad y un poco menos de la cuarta parte dos actividades. Probablemente el hecho de pertenecer a un grupo de participación social resulta beneficioso para realizar actividades físicas por la relación positiva con el medio que los rodea y el afecto motivacional. La evidencia científica menciona que la interacción y participación es fundamental y favorece la calidad de vida ${ }^{19}$.

Las actividades domésticas son consideradas como parte de la vida diaria de las personas, especialmente en el caso de las mujeres, por lo que elegir este tipo de actividades en una casa club donde existe otras actividades recreativas es de llamar la atención, ya que más de un cuarto de los participantes menciono realizar la tarea de cocina/repostería. Posiblemente el hecho de compartir, aprender, y tener el placer a este respecto motiva a considerar esta actividad como recreativa por el dominio que aparentemente pueden poseer algunas de las participantes. No se 
Salazar-Barajas ME, Huerta-Gallegos MV, Lankenau-Fuentes FI, Carmona-Carmona R, Duran-Badillo T,

encontró un estudio que hablara de la satisfacción de esta tarea con sus iguales, sin embargo se ha comprobado que compartir tarea domesticas es un elemento clave para la adquisición de mayores grados de autonomía 20 .

El apoyo social implica percibir las muestras de ayuda, afecto y confianza por parte de familiares y amigos, al respecto el apoyo social percibido de confianza y afectivo se pudo observar en más de la mitad de los participantes. Los resultados reportados confirman que "contar con personas que se preocupan por lo que me pasa" y "hablar con alguien sobre problemas familiares $y$ personales" es de gran relevancia para los adultos mayores y "el recibir amor y afecto" es indispensable para ellos. Estos resultados son contrarios a lo reportado en un estudio ${ }^{21}$ donde los adultos mayores experimentaron escaso apoyo social afectivo. Sin embargo, se pudo observar que más de tres cuartos de los participantes no recibieron en invitaciones para socializar, lo cual puede generar sentimientos de abandono emocional $y$ repercutir cognitivamente por la sensación de baja autoestima.

El apoyo social presento correlación positiva con las actividades recreativas una relación que no ha sido documentada por otros estudios, sin embargo si se conoce el efecto benéfico con el afrontamiento al envejecimiento y sobre los síntomas depresivos ${ }^{22}$.

Un estudio brasileño sugiere una mayor atención a los programas de ejercicio físico y la interacción/socialización de la comunidad, con el objetivo de alcanzar problemas de motivación, preferiblemente que puedan cubrir los beneficios biopsicosociales tanto para mujeres como para hombres mayores, con la recuperación de su autoestima y confianza en sí mismos. contra los estereotipos naturalizados por la sociedad $^{23}$.

\section{CONCLUSIÓN}

Los resultados obtenidos de esta investigación coinciden con otros hallazgos reportados en la literatura científica, sin embargo, se puede concluir que profundizar 
Salazar-Barajas ME, Huerta-Gallegos MV, Lankenau-Fuentes FI, Carmona-Carmona R, Duran-Badillo T,

en estas variables apoyo social $y$ actividades recreativas en su conjunto sería beneficioso para este grupo poblacional. Los adultos mayores son considerados vulnerables y por consiguiente continuar con el estudio de estas temáticas y sus relaciones posiblemente se pueda contribuir a mejorar la calidad de vida de los adultos.

$$
\text { El involucramiento }
$$

familiar, amigos y otras personas significativas durante la vejez, son de suma importancia para los adultos mayores y esta responsabilidad recae en el involucramiento de toda la sociedad, las instituciones educativas y la familia. Para la disciplina de enfermería continua siendo un reto la educación para generar prácticas que beneficien a la población especialmente al adulto mayor.

No se realizó diferencias por género ya que la mayoría de la muestra fueron mujeres, posiblemente la percepción del apoyo familiar en esta muestra este influida por personas que comparten la misma red social.

\section{REFERENCIAS}

1. Organización Mundial de la Salud. Envejecimiento y ciclo de vida; 2019 [acceso en 2019 agosto 24] Disponible en: https: / / www. who.int/ageing/ab out/facts/es/.

2. Instituto Nacional de Estadística y Geografía. Análisis prospectivo de la población de 60 años de edad en adelante; 2017 [acceso en 2019 agosto 24] Disponible en:

https: / /www.gob.mx/cms/uploa ds/attachment/file/201801/An_l isis_prospectivo_de_la_poblaci_n _de_60_a_os_en_adelante.pdf.

3. Ramos Monteagudo AM, Yordi García M, Miranda Ramos MA. El envejecimiento activo: importancia de su promoción para sociedades envejecidas. AMC. 2016; 20(3):330-337.

4. Instituto Nacional de Geriatría. Envejecimiento. 2017 [acceso en 2020 febrero 26] Disponible en: http: / /www.geriatria.salud.gob. $\mathrm{mx} /$ contenidos/institucional/env ejecimiento.html.

5. Gutiérrez Robledo LM, Agudelo Botero M, Giraldo Rodríguez L, Medina Campos RH. Hechos y desafíos para un envejecimiento 
Salazar-Barajas ME, Huerta-Gallegos MV, Lankenau-Fuentes FI, Carmona-Carmona R, Duran-Badillo T,

saludable en México. 2016.

Disponible

en:

http: / /www.geriatria.salud.gob.

$\mathrm{mx} /$ descargas/publicaciones/hec hos-desafios.pdf

6. Triadó TC. Envejecimiento activo, generatividad y aprendizaje. Aula abierta. 2018; 47(1):63-66.

7. Cardona $A D$, Segura CÁ, Segura CA, Muñoz R D, Agudelo C MC. La felicidad como predictor de funcionalidad familiar del adulto mayor en tres ciudades de Colombia. Hacia Promoc Salud. 2018; 24(1):97-111.

8. Jiménez AB, Baillet Esquivel LE, Ávalos- PF, Campos AL. Dependencia funcional y percepción de apoyo familiar en el adulto mayor. Aten Fam. 2016; 23(4):129-133.

9. Figueroa LA, Soto Carballo D, Santos Fernández NA. Calidad de vida y apoyo social percibido en adultos mayores. Rev Cienc Méd. 2016; 20(1).

10. Calderón-Mafud JL, LacaArocena F, Pedroza-Cabrera FJ, Pando-Moreno M. Efecto de la socialización organizacional sobre el compromiso organizacional en trabajadores mexicanos. Rev Electrónica Psicol Iztacala. 2015; 18(3):1011-1034.

11. Morales S, Díaz T, Cumbajin M, Torres A, Analuiza E. Influencia de las actividades físicorecreativas en la autoestima del adulto mayor. Rev Cub de Invest Biomed. 2016; 35(4):366374.

12. Garza Sanchez RI, Nuñez Udave LF, Bruno F, Castro Saucedo LK, Acosta Ramírez MF. La autodeterminación del adulto mayor una comparación entre México y Chile. CNEIP. 2019; $\mathrm{s} / \mathrm{n}: 1-9$.

13. Broadhead WE, Gehlbach SH, Degruy FV, Kaplan BH. The Duke-UNC functional social support questionnaire: Measurement for social support in family medicine patients. Med Care. 1998; 26(2):709-723.

14. Piña LA, Rivera IB. Validación del cuestionario de apoyo social funcional en personas seropositivas al VIH del noroeste de México. Cienc Enferm. 2007; 13(1):53-63.

15. Hernández Sampieri R, Fernández Collado C, Baptista Lucio MP. Metodología de la 
Salazar-Barajas ME, Huerta-Gallegos MV, Lankenau-Fuentes FI, Carmona-Carmona R, Duran-Badillo T,

investigación. 6ed. México. McGrawHill; 2010.

16. Sánchez-Padilla LM, GonzálezPérez U, Alerm-González A, Bacallao-Gallestey J. Calidad de vida psíquica y estado de salud física en el adulto mayor. Rev haban cienc méd. 2014; 13(2):337-349.

17. Instituto Nacional de Estadística y Geografía. Educación. México: INEGI; 2018. Disponible: cuéntame.inegi.org.mx/monogr afías/información/df/población /educación/educación.aspx.

18. Organización Mundial de la Salud (OMS). Actividad Física. 2018. Disponible: https://www.who.int/es/newsroom/factsheets/detail/physical-activity

19. Brown TC, Fry MD. Effects of an Intervention with a University Recreation Center Staff to Foster a Caring, Task-Involving Climate. J Clin Sport Psychol. 2015; 9(1):59-67.

20. García González AJ, Froment F. Beneficios de la actividad física sobre la autoestima y la calidad de vida de las personas mayores. Retos. 2018; 33:3-9.

21. Torres-Palma WI, Flores-Galaz MM. Factores predictores del bienestar subjetivo en adultos mayores. Rev Psicol (Lima). 2018; 36(1):9-48.

22. Salazar-Barajas ME, LilloCrespo M, Hernández-Cortez PL, Villarreal-Reyna MA, Gallegos-Cabriales EC, GómezMesa MV, et al. Factores que contribuyen al envejecimiento activo en los adultos mayores, desde el marco del Modelo de Adaptación de Roy. Invest educ enferm. 2018; 36(2):e08.

23. França CBS, Barbosa RFM, Fett WCR, Fett CA. Exercício físico e envelhecimento: a percepção de idosas quanto à imagem corporal. J Health NPEPS. 2016; 1(1):94-108.

Conflito de interesses: Os autores declaram não haver conflito de interesses. 
Salazar-Barajas ME, Huerta-Gallegos MV, Lankenau-Fuentes FI, Carmona-Carmona R, Duran-Badillo T, Ruíz-Cerino JM, et al.

Apoyo social y participación en actividades recreativas...

\section{Participação dos autores:}

- Concepção: Salazar-Barajas ME, Huerta-Gallegos MV, Lankenau-Fuentes FI, Carmona-Carmona R, Duran-Badillo T, Ruíz-Cerino JM, Pérez-Zúñiga $X$, Guerra-Ordóñez JA.

- Desenvolvimento: Salazar-Barajas ME, Huerta-Gallegos MV, Lankenau-Fuentes FI, Carmona-Carmona R, Duran-Badillo T, Ruíz-Cerino JM, Pérez-Zúñiga $X$, Guerra-Ordóñez JA.

- Redação e revisão: Salazar-Barajas ME, Huerta-Gallegos MV, Lankenau-Fuentes FI, Carmona-Carmona R, Duran-Badillo T, Ruíz-Cerino JM, Pérez-Zúñiga $X$, Guerra-Ordóñez JA.

Como citar este artigo: Salazar-Barajas ME, HuertaGallegos MV, Lankenau-Fuentes FI, Carmona-Carmona R, Duran-Badillo T, Ruíz-Cerino JM, et al. Apoyo social y participación en actividades recreativas en adultos mayores fronterizos. J Health NPEPS. 2020; 5(1):68-83.

Submissão: 03/02/2020

Aceito: $23 / 05 / 2020$

Publicado: 01/06/2020 